Research Article

\title{
Deep Learning-Based Self-Efficacy X-Ray Images in the Evaluation of Rheumatoid Arthritis Combined with Osteoporosis Nursing
}

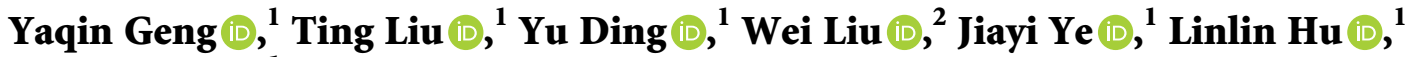 \\ and Li Ruan (iD) \\ ${ }^{1}$ Department of Rheumatology, The Affiliated Changzhou No. 2 People's Hospital of Nanjing Medical University, Changzhou, \\ Jiangsu 213003, China \\ ${ }^{2}$ Department of Image, The Affiliated Changzhou No. 2 People's Hospital of Nanjing Medical University, Changzhou, \\ Jiangsu 213003, China
}

Correspondence should be addressed to Li Ruan; gengyq888@njmu.edu.cn

Received 29 July 2021; Revised 29 September 2021; Accepted 30 September 2021; Published 26 October 2021

Academic Editor: Gustavo Ramirez

Copyright (C) 2021 Yaqin Geng et al. This is an open access article distributed under the Creative Commons Attribution License, which permits unrestricted use, distribution, and reproduction in any medium, provided the original work is properly cited.

To explore the application of self-efficacy in X-ray image analysis based on deep convolutional neural network (DCNN) in the care and treatment of osteoporosis patients with rheumatoid arthritis. In this study, 90 patients with osteoporosis were divided into the control group and the experimental group for DCNN combined with X-ray diagnosis. Patients in the control group were given routine nursing care, and those in the experimental group were given comprehensive nursing care. The bone mineral content, selfefficacy, anxiety, and depression in the femur and lumbar spine after care were compared. The results showed that the accuracy, sensitivity, and false-negative rate of X-ray image recognition of osteoporosis based on DCNN were $91 \%$, 98\%, and $2 \%$, respectively. The bone mineral contents of femur and lumbar vertebra in the experimental group were significantly higher than those in the control group $(P<0.05)$. The anxiety, depression, and self-efficacy scores of patients in the experimental group were significantly higher than those in the control group $(P<0.05)$. In conclusion, the accuracy rate of DCNN combined with X-ray plain film imaging in the detection of osteoporosis is high. Comprehensive nursing intervention can improve the curative effect and self-efficacy of patients. The improvement of self-efficacy is a related factor for the improvement of patients' negative emotions and quality of life.

\section{Introduction}

In recent years, computer technology has been widely used in the medical field, such as computed tomography (CT) and $\mathrm{X}$-ray imaging technology [1]. Different tissues can absorb different number of rays. Based on this principle, the technology displays three-dimensional images of internal structure of the human body, which provides convenience and basis for clinical diagnosis [2]. CNN is a kind of feedforward neural network, which can effectively reduce the complexity of the feedback neural network. It can identify some distorted but non-deformed two-dimensional images, such as those with displacement, scaling, etc., and is one of the representative algorithms of deep learning $[3,4]$. In addition, neural network recognition technology is widely used in image segmentation [5]. Neural network has a huge number of connections, which is easy to include spatial information, and can better solve the problems of unevenness and noise in image recognition $[6,7]$. The first convolutional neural network is a time delay network proposed by foreign scholars and experts, which belongs to the convolutional neural network for speech recognition. Then, the translational invariant artificial neural network has been successively proposed and applied to medical image detection and recognition $[8,9]$. Later, with the advancement of deep learning theory and the update of numerical computing equipment, the representation learning ability of convolutional neural networks has attracted extensive attention from all walks of life [10]. 
Osteoporosis is a systemic bone disease, which is mainly characterized by an increased incidence of fractures, decreased bone mineral density, and structural damage. The clinical manifestations of osteoporosis are often deformation or fracture of the spine, limited movement, or difficulty in walking $[10,11]$. According to the etiology, it can be divided into primary and secondary osteoporosis. Secondary osteoporosis is mainly caused by secretary metabolic diseases such as hyperthyroidism or the influence of drugs and hormones. The fracture and lesion at the lesion site can be found through $\mathrm{X}$-ray plain film diagnosis of the lesion site $[12,13]$. Rheumatoid arthritis (RA), an autoimmune disease, has swelling and pain in the joints in the early stage of its occurrence, but then, with the development of the disease, a large amount of calcium substances is lost, resulting in bone changes and even osteoporosis, which increases the difficulty in clinical diagnosis and treatment $[14,15]$.

In this study, 90 patients with rheumatoid arthritis combined with osteoporosis were selected as the research object, and the DCNN combined with X-ray plain film diagnosis was conducted. They were divided into the control group and the experimental group, which were given routine nursing care and comprehensive nursing intervention, respectively. After care, the bone mineral content in the femur and lumbar vertebra, self-efficacy, anxiety, and depression were compared between the two groups, and the quality of life of the patients was evaluated according to the summary table of quality of life measurement. Thus, the application of self-efficacy in the care and evaluation of osteoporosis in rheumatoid arthritis was studied based on $\mathrm{X}$-ray image analysis of convolutional neural network in-depth learning.

\section{Materials and Methods}

2.1. General Information. In the study, 90 patients with RA combined with osteoporosis, admitted to our hospital from September 2018 to September 2020, were selected as the research subjects (48 males and 42 females). They were enrolled into a control group and an experimental group, each with 45 cases, and all accepted DCNN-based X-ray plain film examinations. The control group had routine nursing, and the experimental group had comprehensive nursing including social support, psychological counseling, daily exercise guidance, rational medication, and emotional relief. The nursing effects were compared for the bone mineral content in the femur and lumbar spine, self-efficacy scores, and anxiety and depression scores, and the quality of life was assessed. The study has been approved by the Ethics Committee of the hospital. The patients and their families were informed of the study and had signed the consent forms.

Inclusion criteria were as follows: (i) patients between 40 and 60 years old; (ii) patients with clear consciousness who can cooperate with doctors in treatment and sample collection; (iii) patients with RA and osteoporosis confirmed by premenstrual examination; (iv) patients with complete clinical data and information; and (v) patients with no history of mental illness.
Exclusion criteria were as follows: (i) patients who withdrew and transferred for treatment due to personal reasons; (ii) patients with other serious diseases or infectious diseases; (iii) patients with incomplete case data; (iv) patients with severe arthritis or patients who had undergone treatment for similar conditions; (v) patients who had other system or organ diseases; and (vi) patients who did not accept treatment.

2.2. X-Ray Plain Film Diagnosis. The X-ray plain film on the lesions of RA was taken for osteoporosis diagnosis. After checking the basic information, the examiner determined the specific body part to diagnose, selected the appropriate size of the film according to the actual size of the inspection site, removed the objects that can affect the irradiation effect at the inspection site, and adjusted the voltage, current, and time for preliminary preparation.

2.3. DCNN Learning Algorithm. In the study, the DCNN learning algorithm is applied to recognize the X-ray plain film of patients with RA combined with osteoporosis. In the image segmentation, automatic learning features of network parameters are automatically adjusted by supervised learning, and the image processing process is shown in Figure 1.

To solve the problem that the edge of the lesion in the image is not clear and the local contrast is insufficient, the adaptive histogram equalization method is used to enhance the image contrast, which is defined as follows:

$$
f(n)=\frac{255 \times C(n)}{X * X} .
$$

The size of the sliding window is defined as $X * X, f(n)$ is the local mapping function, and $C(n)$ is the cumulative distribution function. Then, the slope of the local mapping function $\mathrm{f}(\mathrm{n})$ is calculated as follows:

$$
\begin{aligned}
K & =\frac{\mathrm{d}(f(n))}{\mathrm{d} i} \\
& =H E(n) \times \frac{255}{X * X} .
\end{aligned}
$$

When the maximum local mapping function slope is $K_{\max }$, the maximum histogram height is $L_{\max }$, which is calculated as follows:

$$
\begin{aligned}
L_{\max } & =K_{\max } \times \frac{X * X}{255}, \\
O_{n} & =T\left(\sum_{m=1}^{t} Q_{n m} I_{n}-\gamma_{n}\right) .
\end{aligned}
$$

The calculation model of neural network is as (4), where $O_{n}$ is the output of the neuron, $I_{n}$ ( $\mathrm{n}$ is $1,2,3, \ldots, \mathrm{n}$ ) is the input signal, $T$ is the neuron activation function, $Q_{n m}$ indicates the connection strength between two neurons, and $\gamma_{n}$ indicates the threshold of activating neurons. 


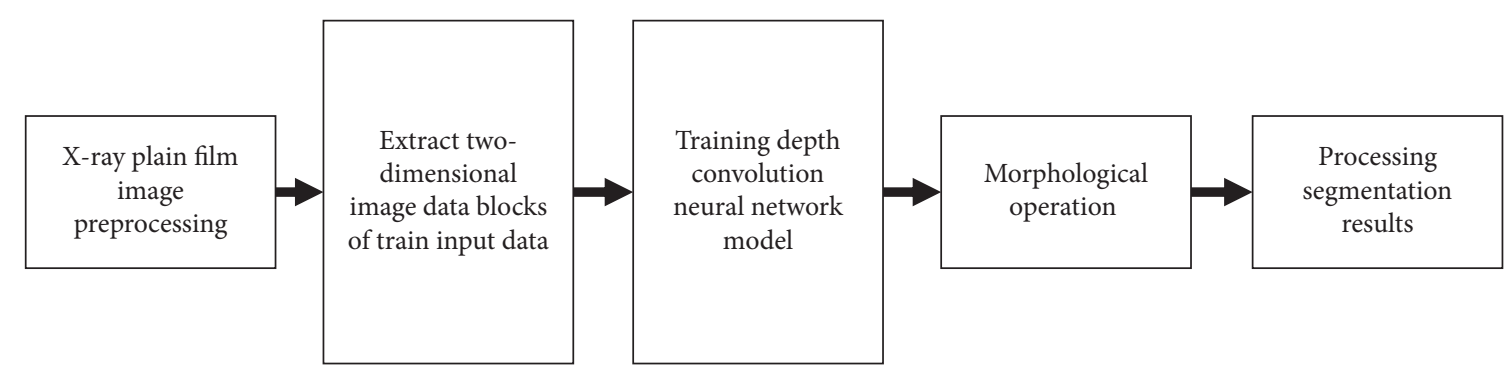

FIgURE 1: The X-ray plain film image processing based on DCNN learning algorithm.

$$
\mathrm{Wei}(T, F)=\sum_{n=1}^{m} W_{n} f\left(T_{n}, F_{n}\right)
$$

The severity of rheumatoid arthritis is evaluated through $\mathrm{X}$-ray image data, and all levels of weight values are assigned through a weighted loss function. The weight of loss is calculated as equation (5). Among them, $n$ is the sample size, $T$ is the real value input, $F$ is the predicted value input, and $f$ represents the loss function. In general, CNN algorithm mainly refers to a linear operation method for grid data, including several basic simple operation processes. Compared with other neural networks, the CNN has independent connection and calculation methods, but they are all based on the backpropagation algorithm, and the network parameters are adjusted and optimized on this basis.

$$
T(n)=\int_{-\infty}^{+\infty} I(x) O(n-x) Y_{x},
$$

where $I(x)$ and $O(x)$ are integral functions, $I(x)$ represents the input, $O(x)$ represents the convolutional kernel, and $T(n)$ is the feature mapping, which is expressed as follows:

$$
T(n)=\sum_{x=-\infty}^{+\infty} I(x) O(n-x) .
$$

In practice, for discrete data in the image, it is defined that $I(x)$ and $O(x)$ are both discrete variables. Then, the convolution of $I(x)$ and $O(x)$ is expressed as equation (7). CNN can recognize images by sliding window, selective search, and other methods. First, it can judge whether the window is the target and defines it as the regression problem of the occurrence probability of each target in image segmentation.

2.4. Evaluation Indicators and Standards. Patients accepted routine nursing and comprehensive nursing, including social support, psychological counseling, daily exercise guidance, rational medication, and emotional relief, respectively. The nursing effects were compared for the bone mineral content in the femur and lumbar spine, self-efficacy scores, and anxiety and depression scores, and the quality of life was assessed. Among them, the self-efficacy was assessed according to Table 1.

Dice similarity coefficient (DSC), average pixel accuracy (MPA), and average intersection ratio (MIOU) were used to evaluate the difference of ROI between manual marking by clinicians and automatic marking by algorithms, and the calculation formula was as follows:

$$
\begin{aligned}
\mathrm{DSC} & =\frac{2 \times\left|S \cap S_{0}\right|}{S_{0}+S}, \\
\mathrm{MPA} & =\left(1-\frac{\left|S_{0}-S\right|}{S_{0}}\right), \\
\mathrm{MIOU} & =\frac{S_{0} \cap S}{S_{0} \cup S},
\end{aligned}
$$

where $S_{0}$ represents the ROI manually marked by the clinician and $S$ represents the ROI automatically segmented by the algorithm.

2.5. Statistical Methods. The data were processed by SPSS19.0, the measurement data were calculated as mean\pm standard deviation $(\bar{x} \pm s)$, and the count data were expressed as a percentage (\%). The analysis of variance was adopted for comparison between groups. The difference was statistically significant with $P<0.05$.

\section{Results}

3.1. X-Ray Images of Osteoporosis. Figure 2 shows an X-ray image of the right knee joint of a patient with osteoporosis. It was evident that the bone density of the right knee was decreased, the trabecular bone was thinned and sparse, the space between bone marrow cavity and the trabecular bone was widened, the bone cortex was thinned, and there was stratification. The edge of the knee joint became sharp, the joint space became narrower, and there was millimeter-sized scattered radiolucent space with a blurred boundary. Clear low-density radiolucent areas can be seen above and below the patella.

Figure 3 shows an X-ray image of the ankle joint of a patient with osteoporosis. The joint was saddle-shaped and about $4 \mathrm{~mm}$ wide. The lateral X-ray film found that the joint space appeared as a forward-backward convex curved line, and the inner and outer ankles were unclear because they overlapped the shadow of the talus. The anteroposterior length of the upper articular surface of the talus was much longer than that of the lower articular surface of the tibia.

The accuracy of ROI segmentation by different algorithms was objectively evaluated by DSC, MPA, and MIOU, and two typical algorithms, support vector machine (SVM) 
TABle 1: C-RASE: Chinese version of Rheumatoid Arthritis Self-Efficacy Scale.

\begin{tabular}{|c|c|c|c|c|c|c|c|c|c|}
\hline Items & $\begin{array}{c}\text { Factor } \\
1 \\
\end{array}$ & $\begin{array}{c}\text { Factor } \\
2 \\
\end{array}$ & $\begin{array}{c}\text { Factor } \\
3\end{array}$ & $\begin{array}{c}\text { Factor } \\
4 \\
\end{array}$ & $\begin{array}{c}\text { Factor } \\
5 \\
\end{array}$ & $\begin{array}{c}\text { Factor } \\
6 \\
\end{array}$ & $\begin{array}{c}\text { Factor } \\
7 \\
\end{array}$ & $\begin{array}{c}\text { Factor } \\
8 \\
\end{array}$ & Factor 9 \\
\hline 24. Having daily exercise & 0.904 & 0.027 & 0.074 & 0.119 & 0.008 & 0.038 & 0.028 & 0.010 & 0.156 \\
\hline $\begin{array}{l}\text { 13. Solving the difficulties encountered in daily life } \\
\text { through exercise }\end{array}$ & 0.875 & 0.191 & 0.208 & 0.155 & 0.018 & 0.052 & 0.013 & -0.093 & 0.105 \\
\hline 1. Adopting relaxation therapy to relieve pain & 0.501 & 0.252 & 0.150 & 0.015 & 0.105 & -0.218 & 0.144 & 0.125 & -0.355 \\
\hline 27. Paying close attention to adverse drug reactions & 0.104 & 0.729 & 0.102 & 0.040 & -0.036 & 0.120 & 0.068 & -0.116 & 0.128 \\
\hline 3. Relieving pain by protecting joints & -0.082 & 0.658 & 0.166 & 0.154 & 0.109 & -0.295 & 0.068 & -0.182 & 0.183 \\
\hline $\begin{array}{l}\text { 14. Arranging daily work and clarifying the } \\
\text { problems that need to be solved first }\end{array}$ & 0.138 & 0.600 & -0.166 & -0.007 & 0.044 & 0.033 & 0.305 & 0.126 & -0.094 \\
\hline $\begin{array}{l}\text { 7. Routine hot drinks before going to bed to help } \\
\text { sleep }\end{array}$ & 0.154 & 0.520 & 0.148 & -0.157 & 0.045 & 0.219 & -0.445 & 0.037 & -0.144 \\
\hline 26. Able to learn how to use drugs rationally & 0.335 & 0.444 & 0.156 & 0.113 & 0.006 & 0.166 & 0.069 & 0.206 & -0.112 \\
\hline $\begin{array}{l}\text { 23. Relaxing oneself by listening to music or through } \\
\text { other soothing methods }\end{array}$ & 0.036 & 0.217 & 0.797 & 0.082 & 0.154 & 0.042 & -0.080 & 0.109 & -0.105 \\
\hline axed mind to deal with anxiety & 0.143 & 0.067 & 0.710 & 0.115 & 0.033 & -0.076 & 0.181 & 0.234 & 0.201 \\
\hline 22. Relie & 0.284 & -0.001 & 0.675 & 0.204 & -0.143 & 0.109 & 0.094 & 0.032 & 0.009 \\
\hline ergy for leisure activities, hobbies, or & 0.146 & 0.065 & 0.160 & 0.882 & 0.017 & 0.059 & -0.041 & -0.006 & 0.046 \\
\hline $\begin{array}{l}\text { time for leisure activities, amateur } \\
\text { ocial activities }\end{array}$ & 0.139 & 0.031 & 0.188 & 0.872 & 0.071 & -0.035 & -0.072 & -0.025 & .032 \\
\hline 11. Using auxiliary tools for help & -0.008 & 0.107 & 0.332 & 441 & -0.074 & 0.220 & 0.139 & 0.337 & .312 \\
\hline $\begin{array}{l}\text { 8. Relaxing before going to bed to improve sleep } \\
\text { quality }\end{array}$ & 07 & 0.048 & 0.007 & 0 & 0. & 4 & 9 & 99 & -0.038 \\
\hline 6. Relaxing before going to bed to have a deep sleep & 0.017 & 0.032 & .046 & -0 & 0.905 & 0.109 & -0.064 & 0.050 & 0.043 \\
\hline $\begin{array}{l}\text { 12. Seeking help from relatives and friends in time } \\
\text { when there are difficulties in daily life or work }\end{array}$ & -0.00 & 0.0 & 00 & $0 \Omega 4$ & 0.012 & 00 & 0.112 & -0.047 & 0.0 \\
\hline $\begin{array}{l}\text { 16. Explaining to relatives and friends if you need } \\
\text { help }\end{array}$ & 0.053 & 0.096 & 0.035 & 0.027 & 0.195 & 0.750 & -0.008 & -0.070 & -0.100 \\
\hline 28. Seeking help when feeling down & -0.081 & 0.130 & -0.016 & -0.035 & -0.253 & 0.486 & -0.198 & 0.444 & 0.240 \\
\hline $\begin{array}{l}\text { 10. Accepting that fatigue is one of the symptoms of } \\
\text { RA }\end{array}$ & 0.144 & 0.091 & 0.001 & -0.087 & -0.183 & 0.102 & 0.806 & 0.030 & -0.087 \\
\hline $\begin{array}{l}\text { 9. Knowing that fatigue is caused by arthritis and } \\
\text { adjusting oneself appropriately }\end{array}$ & -000 & 0.261 & 0.232 & -0 & - & 0.032 & 0.686 & 0.037 & 0.220 \\
\hline 4. Maintaining a positive attitude towa & 0.065 & -0.179 & 0.314 & -0.013 & 0.135 & -0.032 & -0.108 & 0.771 & -0.068 \\
\hline 20. Being positive when feeling down & -0.038 & 0.060 & 0.106 & 0.028 & & -0.139 & 0.293 & 0.669 & 0.296 \\
\hline ttention to ease the pain & 0.327 & -0.006 & 0.130 & 0.216 & -0.001 & -0.050 & -0.018 & 0.051 & 0.638 \\
\hline $\begin{array}{l}25 \text {. Able to control the degree of exercise to avoiding } \\
\text { sports injuries }\end{array}$ & 0.066 & 0.170 & -0.071 & -0.227 & 0.019 & -0.122 & 0.287 & 0.257 & 0.576 \\
\hline
\end{tabular}

Note. Table 1 was derived from the preliminary analysis of the reliability and validity of the Chinese version of the Rheumatoid Arthritis Self-Efficacy Scale (2019).

and Naive Bayes (NB), are introduced for comparison. The results showed that the DSC, MPA, and MIOU values of $\mathrm{CNN}$ algorithm were $0.9410,0.9228$, and 0.8968 , respectively, which were significantly better than those of SVM algorithm and NB algorithm (Figure 4).

3.2. The Incidence of Osteoporosis. Figure 5 presents different locations of osteoporosis and the corresponding number of patients. It was evident that there are more patients with femoral osteoporosis vs. patients with lumbar osteoporosis. There was no notable difference in the number of patients with osteoporosis in different parts between the two groups $(P<0.05)$.

3.3. The Detection Rate of the X-Ray Image. It was evident from Figure 6 that the accuracy and sensitivity of DCNN-based image analysis in detecting osteoporosis were $91.2 \%$ and $98.3 \%$, respectively, and the false-negative rate was only $2 \%$.
3.4. The Anxiety and Depression Scores. Figures 7 and 8 present the proportion of patients with the four mental states of mild depression, anxiety, general, and good. The experimental group had a higher proportion of patients with a good mental state $(62 \%$ vs. $10 \%)(P<0.05)$ and a lower proportion with mild depression ( $3 \%$ vs. $44 \%)$. The patients with anxiety and general mental state accounted for $10 \%$ and $25 \%$, respectively.

3.5. The Bone Mineral Content and Bone Density. Figure 9 shows the bone mineral density index $T$ value after different nursing interventions. It was less than -2.0 in the control group, while it was at a normal level in the experimental group, which was between -1.5 and $-1(P<0.05)$.

3.6. The Self-Efficacy and Exercise Scores. Figure 10 presents the self-efficacy scores after different nursing interventions. It was evident that the self-efficacy score of the 


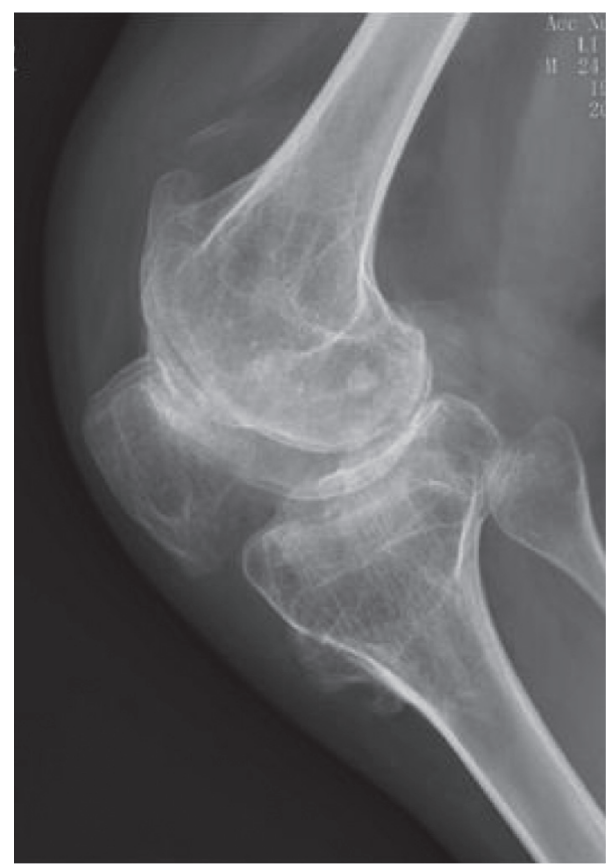

FIgURE 2: The X-ray image of a right knee joint.

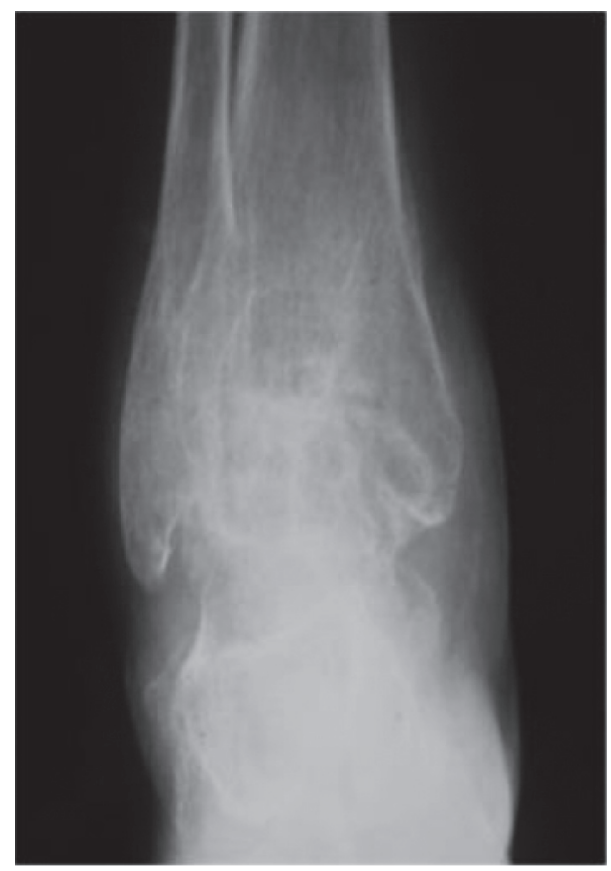

FIgURE 3: The X-ray image of an ankle joint.

experimental group was higher $(78.86 \pm 11.32$ vs. $51.21 \pm 13.37)(P<0.05)$.

Figure 11 shows the exercise status scores after different nursing interventions. It was evident that the exercise status score of the experimental group was higher $(83.81 \pm 13.28$ vs. $60.21 \pm 20.15)(P<0.05)$.

Figure 12 shows the Tampa Scale of Kinesiophobia (TSK) scores of the two groups. It was evident that the TSK score of the experimental group was lower $(28.09 \pm 3.21$ vs. $47.85 \pm 4.88)(P<0.05)$.

\section{Discussion}

Generally, patients with osteoporosis have no obvious symptoms in the early stage, and the disease has developed to skeletal deformities, abnormal lesions, or even fractures when it is finally discovered. X-ray plain films enable the doctor to observe local lesion changes (such as osteoarthritis, intervertebral disc disease, and spine migration). The X-ray image of osteoporosis shows an increase in bone transparency, a decrease in bone trabeculae, and a widening gap. 


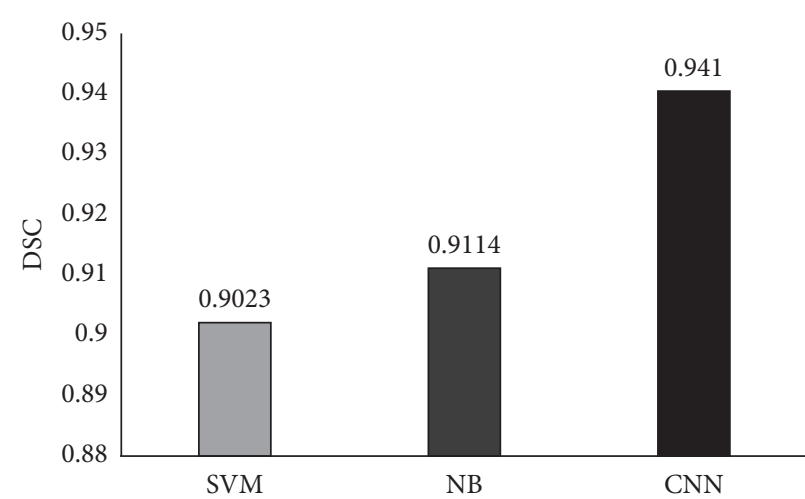

(a)

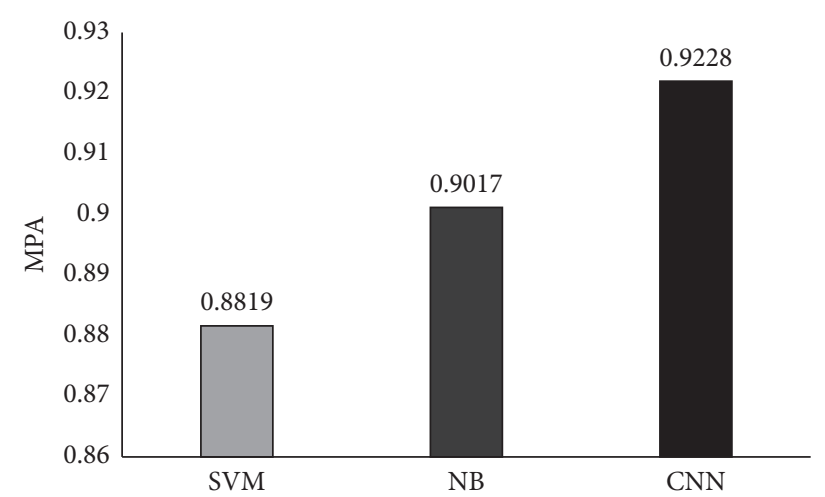

(b)

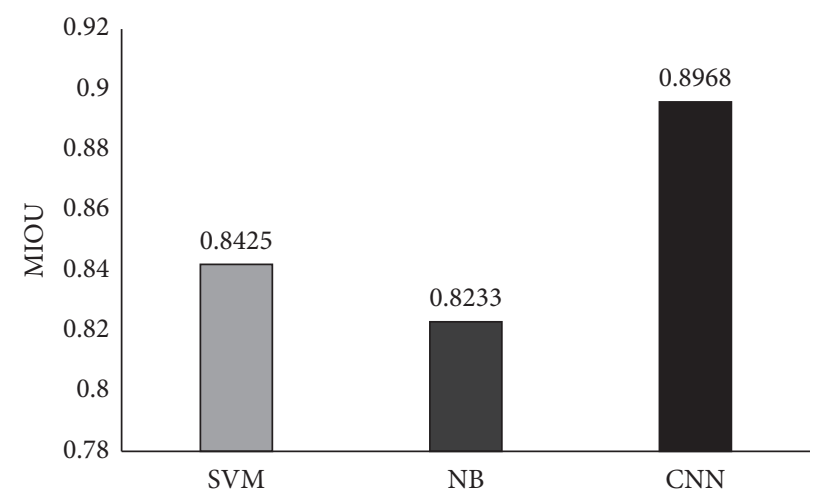

(c)

Figure 4: Evaluation of ROI segmentation results based on different algorithms.

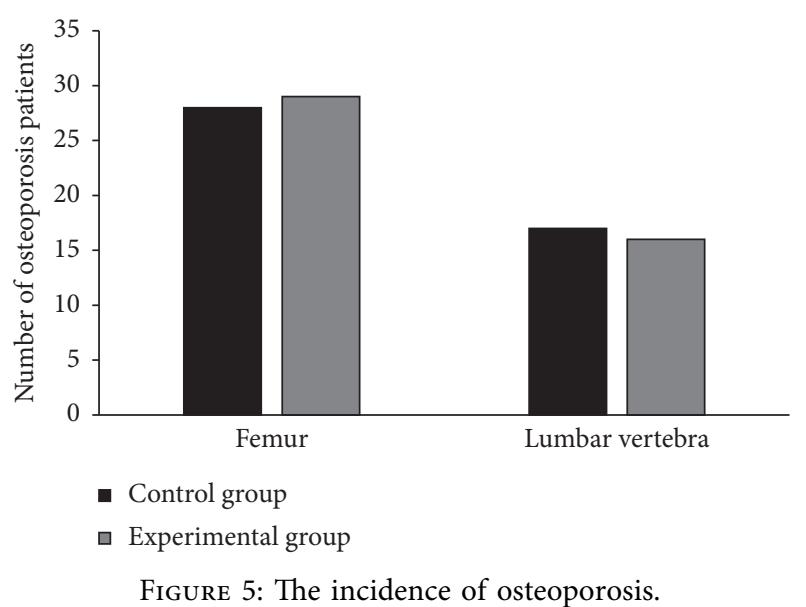

But there are some limitations because the disease will not be discovered until the bone mass is reduced by more than $30 \%$ $[16,17]$. The World Health Organization's classification of osteoporosis in the last century stipulates that when the bone density of adult healthy people drops more than 2.5 from the normal value, it can be judged as osteoporosis [18]. Diseases including hyperparathyroidism, multiple myeloma, and osteomalacia can lead to secondary osteoporosis $[19,20]$. In this study, patients with RA combined with osteoporosis were selected as the research subjects for DCNN-based X-ray plain film examinations. They were given routine nursing

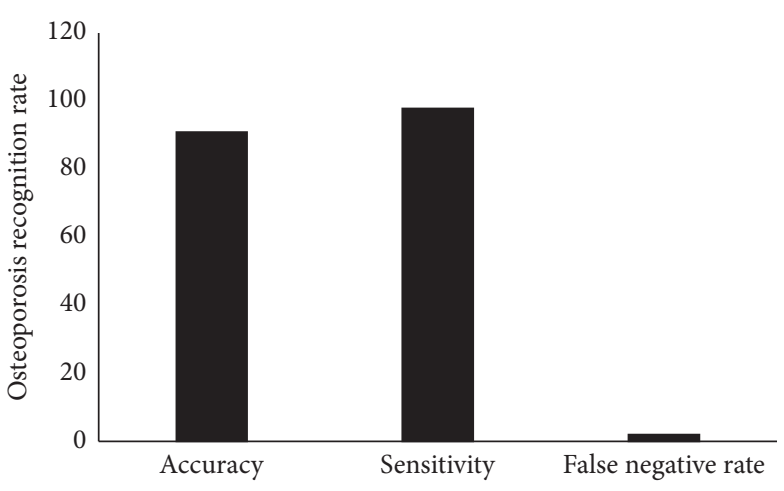

FIgURE 6: Osteoporosis detection rate.

intervention and comprehensive nursing intervention, respectively. The nursing effects were compared for the bone mineral content in the femur and lumbar spine, self-efficacy scores, and anxiety and depression scores. Furthermore, the quality of life was assessed. It was found that in the experimental group, the bone mineral density $T$ value and the self-efficacy score were higher $(P<0.05)$, and the $T$ value was at a normal level. This was consistent with the research results of Picha et al. [21], indicating that DCNN-based $\mathrm{X}$-ray plain film examination has high accuracy in detecting RA combined with osteoporosis and comprehensive nursing such as social support and exercise guidance can improve patients' clinical efficacy, positive emotions, and self-efficacy. 


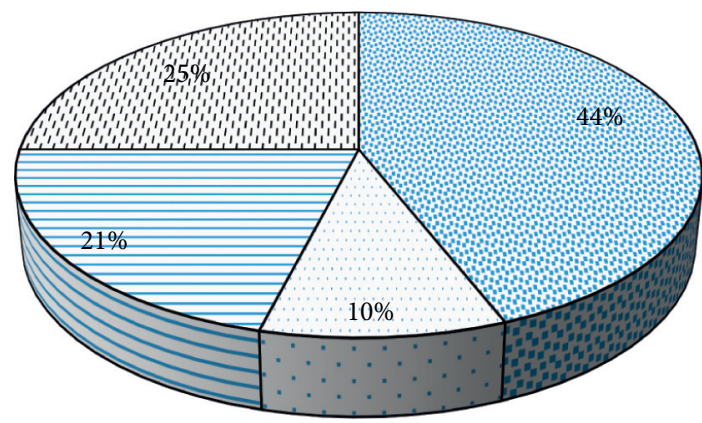

FIgURE 7: The anxiety and depression proportions in the control group.

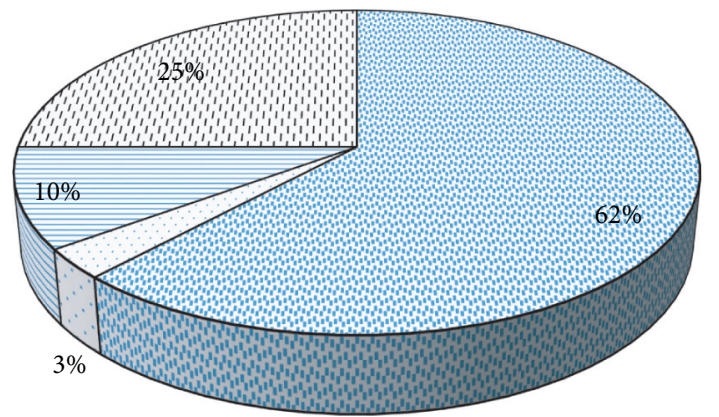

FIgURE 8: The anxiety and depression proportions in the experimental group.

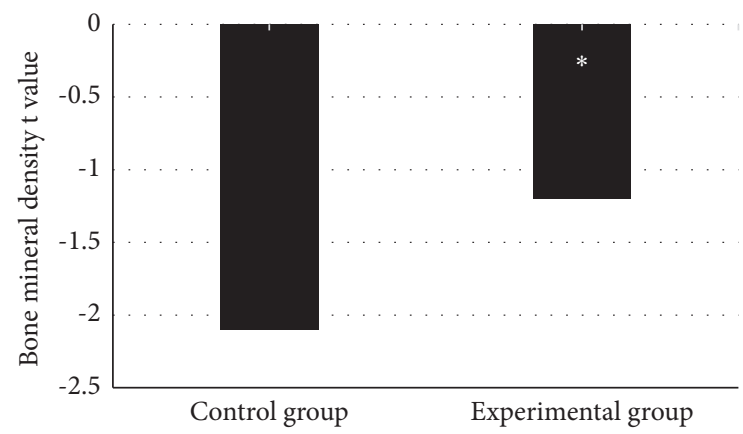

Figure 9: Comparison of bone mineral density $T$ value.

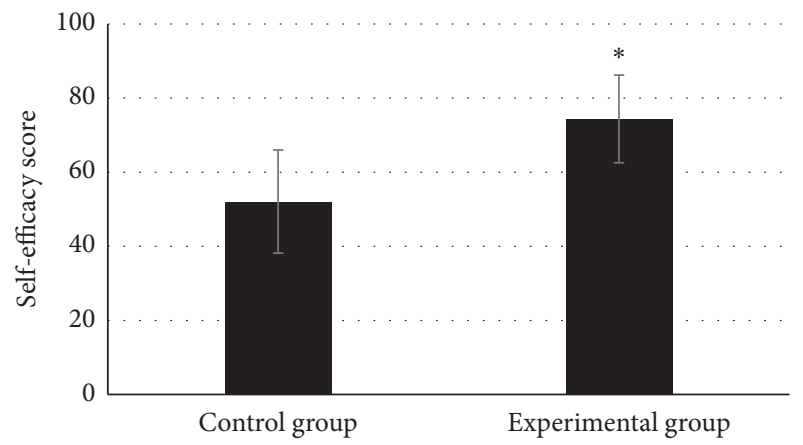

Figure 10: Comparison of self-efficacy scores.

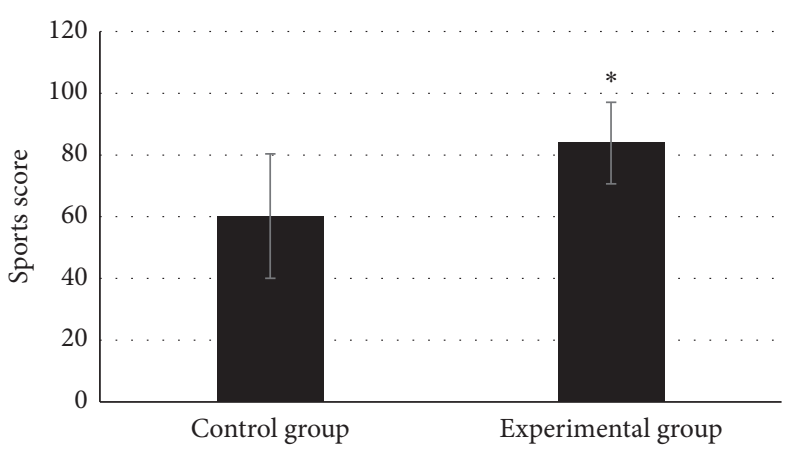

Figure 11: The comparison of exercise scores.

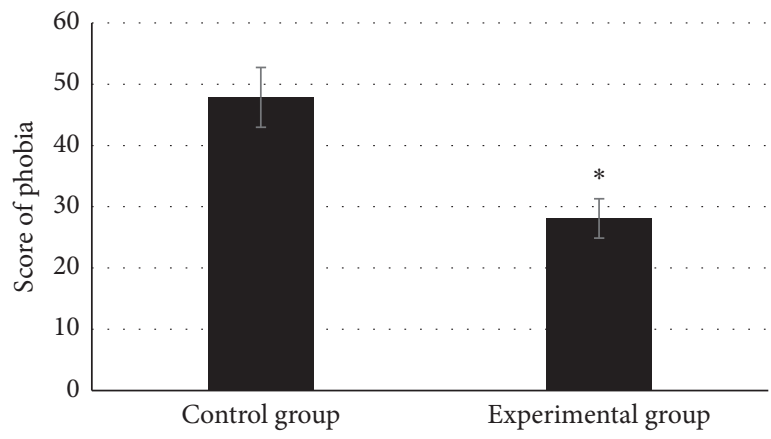

FIgURE 12: Comparison of TSK scores.

The improvement of self-efficacy is a related factor to alleviate patients' negative emotions and promote the quality of life.

\section{Conclusion}

In this study, patients with rheumatoid arthritis and osteoporosis were selected as the research objects and were diagnosed by DCNN combined with X-ray plain film. Routine nursing intervention and comprehensive nursing intervention were given, respectively. DCNN combined with X-ray plain film images can effectively detect rheumatoid arthritis complicated with osteoporosis, with high accuracy. Comprehensive nursing, such as social support and exercise guidance, can improve the clinical efficacy, positive emotion, and self-efficacy of patients. The improvement of self-efficacy is the related factor of patients' negative emotions and improvement of quality of life. However, the sample size selected in this study is small, which may have a certain impact on the experimental results. Therefore, in the subsequent experiments, the sample size will be increased to further study the application of X-ray image analysis of self-efficacy based on deep learning in the nursing and evaluation of rheumatoid arthritis and osteoporosis. In a word, this study provides data support and theoretical basis for the diagnosis and treatment of rheumatoid arthritis complicated with osteoporosis.

\section{Data Availability}

The data used to support the findings of this study are available from the corresponding author upon request. 


\section{Conflicts of Interest}

The authors declare that they have no conflicts of interest.

\section{Authors' Contributions}

Yaqin Geng and Ting Liu contributed equally to this study.

\section{Acknowledgments}

This study was supported by the Changzhou Science and Technology Program (grant no. CJ20190065).

\section{References}

[1] S. L. Solimeo, V.-T. T. Nguyen, S. W. Edmonds et al., "Sex differences in osteoporosis self-efficacy among communityresiding older adults presenting for DXA," Osteoporosis International, vol. 30, no. 5, pp. 1033-1041, 2019.

[2] A. M. Wasserman, "Diagnosis and management of rheumatoid arthritis," American Family Physician, vol. 84, no. 11, pp. 1245-1252, 2011.

[3] E. A. Littlejohn and S. U. Monrad, "Early diagnosis and treatment of rheumatoid arthritis," Primary Care: Clinics in Office Practice, vol. 45, no. 2, pp. 237-255, 2018.

[4] N. Choudhary, L. K. Bhatt, and K. S. Prabhavalkar, "Experimental animal models for rheumatoid arthritis," Immunopharmacology and Immunotoxicology, vol. 40, no. 3, pp. 193-200, 2018.

[5] Y. Zhuang, Y. Shi, J. Zhang et al., "Neurologic factors in patients with vascular mild cognitive impairment based on fMRI," World Neurosurgery, vol. 149, pp. 461-469, 2021.

[6] G. R. Burmester and J. E. Pope, "Novel treatment strategies in rheumatoid arthritis," The Lancet, vol. 389, no. 10086, pp. 2338-2348, 2017.

[7] D. Assayag, J. S. Lee, and T. E. King, "Rheumatoid arthritis associated interstitial lung disease: a review," Medicina, vol. 74, no. 2, pp. 158-165, 2014.

[8] B. Sahiner, A. Pezeshk, L. M. Hadjiiski et al., "Deep learning in medical imaging and radiation therapy," Medical Physics, vol. 46, no. 1, pp. e1-e36, 2019.

[9] R. Feng, M. Badgeley, J. Mocco, and E. K. Oermann, "Deep learning guided stroke management: a review of clinical applications," Journal of Neurointerventional Surgery, vol. 10, no. 4, pp. 358-362, 2018.

[10] A. Hosny, C. Parmar, T. P. Coroller et al., "Deep learning for lung cancer prognostication: a retrospective multi-cohort radiomics study," PLoS Medicine, vol. 15, no. 11, Article ID e1002711, 2018.

[11] M. Hu, Y. Zhong, S. Xie, H. Lv, and Z. Lv, "Fuzzy system based medical image processing for brain disease prediction," Frontiers in Neuroscience, vol. 15, Article ID 714318, 2021.

[12] J. Wallner, M. Schwaiger, K. Hochegger, C. Gsaxner, W. Zemann, and J. Egger, "A review on multiplatform evaluations of semi-automatic open-source based image segmentation for cranio-maxillofacial surgery," Computer Methods and Programs in Biomedicine, vol. 182, Article ID 105102, 2019.

[13] B. Ibragimov and L. Xing, "Segmentation of organs-at-risks in head and neck CT images using convolutional neural networks," Medical Physics, vol. 44, no. 2, pp. 547-557, 2017.

[14] Y. Li, J. Zhao, Z. Lv, and J. Li, "Medical image fusion method by deep learning," International Journal of Cognitive Computing in Engineering, vol. 2, pp. 21-29, 2021.
[15] F. Tang, S. Liang, T. Zhong et al., "Postoperative glioma segmentation in CT image using deep feature fusion model guided by multi-sequence MRIs," European Radiology, vol. 30, no. 2, pp. 823-832, 2020.

[16] D. M. Black and C. J. Rosen, "Postmenopausal osteoporosis," New England Journal of Medicine, vol. 374, no. 3, pp. 254-262, 2016.

[17] S. H. Tella and J. C. Gallagher, "Prevention and treatment of postmenopausal osteoporosis," The Journal of Steroid Biochemistry and Molecular Biology, vol. 142, pp. 155-170, 2014.

[18] L. A. G. Armas and R. R. Recker, "Pathophysiology of osteoporosis," Endocrinology and Metabolism Clinics of North America, vol. 41, no. 3, pp. 475-486, 2012.

[19] L. F. Baccaro, D. Conde, L. Costa-Paiva, and A. M. PintoNeto, "The epidemiology and management of postmenopausal osteoporosis: a viewpoint from Brazil," Clinical Interventions in Aging, vol. 10, pp. 583-591, 201520.

[20] L. Bandeira, E. M. Lewiecki, and J. P. Bilezikian, "Romosozumab for the treatment of osteoporosis," Expert Opinion on Biological Therapy, vol. 17, no. 2, pp. 255-263, 2017.

[21] K. J. Picha, K. N. Jochimsen, N. R. Heebner et al., "Measurements of self-efficacy in musculoskeletal rehabilitation: a systematic review," Musculoskeletal Care, vol. 16, no. 4, pp. 471-488, 2018. 